\title{
Postobstructive pulmonary edema associated with a substernal goitre
}

\author{
Pang-Yen Liu MD, Ming-Lang Shih MD, Chein-Wen Chen MD
}

A 50-year-old woman had experienced 6 months of mild shortness of breath and noise while breathing. She was obese with a body mass index of 32 . Her primary care physician found a neck mass; results of a serum thyroid function test were normal. Her symptoms remitted spontaneously, and she ignored advice to seek further evaluation. Six months later, she had her first episode of dyspnea with cyanosis, followed by decreased consciousness. When the ambulance arrived, her Glasgow Coma Scale score was 7 and her peripheral oxygen saturation was $60 \%$. A nonrebreather oxygen mask was applied during transport.

On arrival at the emergency department, the patient's respiratory rate was 30 breaths/min, pulse rate was 80 beats/min, blood pressure was 107/61 mm Hg and peripheral oxygen saturation was 76\%; her Glasgow Coma Scale score had deteriorated to 6 . Blood gas analysis revealed $\mathrm{pH}$ 6.7, $\mathrm{PCO}_{2} 99.1 \mathrm{~mm} \mathrm{Hg}, \mathrm{PO}_{2} 91.3 \mathrm{~mm} \mathrm{Hg}$ and $\mathrm{HCO}_{3} 11.0 \mathrm{mmol} / \mathrm{L}$, which was consistent with mixed respiratory acidosis and metabolic acidosis. Physical examination showed diffuse rales and some wheezing. After emergent endotracheal intubation, the patient regained consciousness. The endotracheal tube contained copious amounts of a pink frothy secretion. Brain computed tomography scans were normal. Chest radiography done after intubation showed ground-glass opacities bilaterally, suggestive of pulmonary edema (Figure 1A).

Laboratory test results revealed D-dimer levels up to 10.5 (normal < 0.5) $\mu \mathrm{g} / \mathrm{mL}$. Computed tomography of the chest with contrast indicated no filling defect in the pulmonary arteries. There was a nodule visualized that measured $4.0 \times$ $4.5 \times 7.0 \mathrm{~cm}$ in the right thyroid lobe, with intrathoracic extension (Figure 2). Electrocardiography showed ST segment depressions in leads II, III, aVF, V5 and V6. Further laboratory results were as follows: creatine phosphokinase 527 (normal 26-192) U/L; creatine phosphokinase- myocardial band 40 (normal < 25) U/L and troponin I 1.73 (normal <0.50) $\mu \mathrm{g} / \mathrm{L}$. Echocardiography showed hypokinesia over the anterolateral and basal septum with an ejection fraction of $40 \%$. Coronary arteriography was normal. Results of thyroid function and virus serologic tests were negative.

Subsequent chest radiography, 29 hours after intubation, showed resolution of the interstitial, patchy infiltrations (Figure 1B). A second echocardiogram showed normal systolic function with an ejection fraction of $62 \%$. The endotracheal tube was removed after weaning criteria were met and after a successful spontaneous breathing trial with a T-piece circuit. Two hours after extubation, the patient had a second episode of respiratory distress accompanied by stridor with hypoxemia and altered consciousness. Her condition was unresponsive to intravenous hydrocortisone and bilevel positive airway pressure ventilation. After reintubation, she recovered immediately. The patient underwent a tracheostomy and was transferred to the regular ward. She had a third episode of respiratory distress with desaturation 12 hours after the tracheostomy tube was removed.

We suspected she had compression-related tracheomalacia caused by the substernal goitre. Results of ultrasound-guided fine-needle aspiration cytology of the thyroid nodule were nondiagnostic. The patient underwent a right lobectomy of the thyroid, and the cystic nodule,
Competing interests: None declared.

This article has been peer reviewed.

Correspondence to:

Pang-Yen Liu,

bacteriaaaa@mail.ndmctsgh .edu.tw

CMAJ 2012. DOI:10.1503 /cmaj.120256 
measuring $3.0 \times 4.0 \times 7.0 \mathrm{~cm}$, was excised. On pathologic examination, a $1.5 \mathrm{~cm}$ papillary carcinoma was found incidentally within the excised nodule. The tracheostomy tube was removed 7 days after the surgery, and the patient experienced no further respiratory distress.

\section{Discussion}

Pulmonary edema associated with upper airway obstruction was first described in the literature in 1927, when it was observed that prolonged inspiration against a fixed resistance resulted in pulmonary edema in an experimental dog model. ${ }^{1}$ In 1977, Oswalt and colleagues reported a series of adult cases involving pulmonary edema associated with a laryngeal tumour, strangulation and interrupted hanging. ${ }^{2}$ It has been reported following foreign-body aspiration and the relief of airway obstruction from croup and epiglottitis in children. $^{3}$

There are 2 types of postobstructive pulmonary edema, which were first proposed in $1995 .{ }^{4}$ Type I is associated with forceful inspiratory effort in an acute airway obstruction; causes include laryngospasm after extubation, epiglottitis, croup,
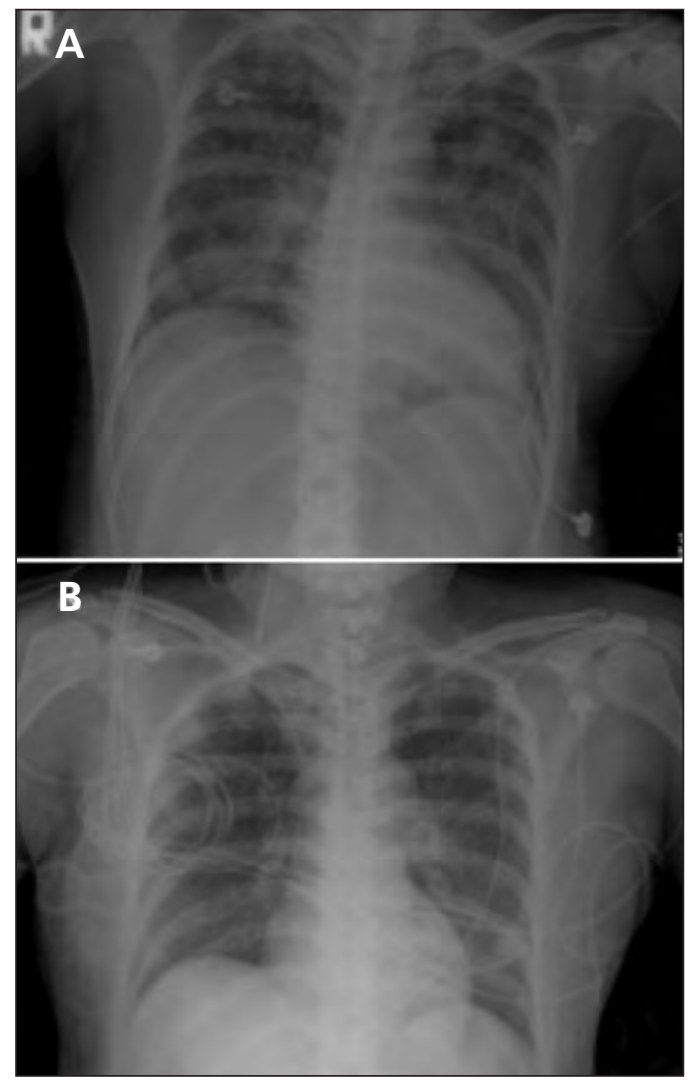

Figure 1: (A) Chest radiograph after intubation in a 50-year-old woman showing bilateral interstitial, patchy infiltrations suggestive of diffuse pulmonary edema. (B) Chest radiograph obtained 29 hours after intubation showing resolution of infiltrations. choking and foreign-body aspiration. Type II occurs after relief of a chronic partial airway obstruction via surgical intervention (Box 1).,5

Subsequent case series, primarily reported by anesthesiologists, indicate that the most common cause is laryngospasm during intubation or extubation. The incidence is as high as 1 in 1000 cases of general anesthesia $(0.094 \%){ }^{6}$ In their case series, Koh and colleagues described postobstructive pulmonary edema occurring in the intensive care unit as an uncommon and lifethreatening condition that is likely underdiagnosed. ${ }^{7}$ The overall incidence of postobstructive pulmonary edema has not been reported.

\section{Why does postobstructive pulmonary edema occur?}

The pathophysiology includes several factors. Inspiration against a closed glottis (modified Müller maneuver) can result in markedly negative intrathoracic pressure. ${ }^{8} \mathrm{~A}$ well-designed study found that these large negative intrathoracic pressures contribute to the hydrostatic mechanisms associated with type I postobstructive pulmonary edema. ${ }^{9}$ Acute upper airway obstruction causes negative intrathoracic pres-

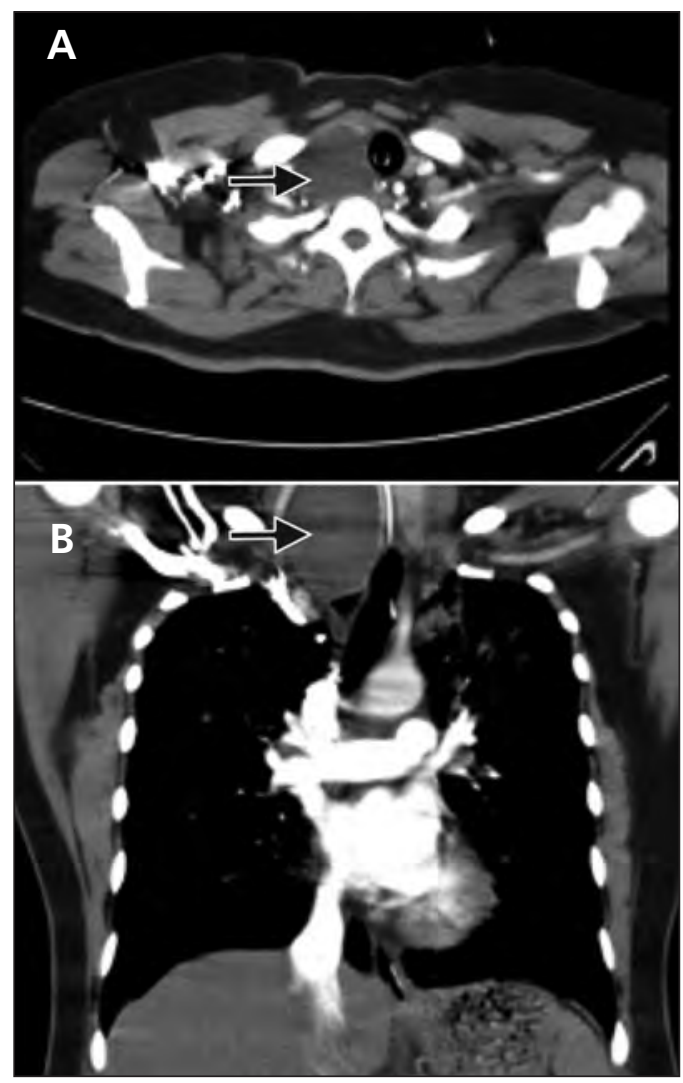

Figure 2: (A) Computed tomographic scan of the chest showing a mass measuring $4.0 \times 4.5 \times 7.0 \mathrm{~cm}$ in the right thyroid, causing the trachea to deviate to the left. (B) Coronal view of the chest showing intrathoracic extension of the thyroid mass. 
sure, hypoxia and a hyperadrenergic state. Increases in the return of blood to the right heart, pulmonary vessel pressure and transudation from the pulmonary capillary space to the interstitium lead to pulmonary edema. Hypoxia and acidosis also depress myocardial contractility, exacerbating pulmonary edema. ${ }^{8,9}$

The pathophysiology of type II postobstructive pulmonary edema is associated with expiration against an upper airway obstruction (Valsalva maneuver) resulting in an increase in intrathoracic pressure. The sudden relief of obstruction causes a decrease in intrathoracic pressure followed by an increase in venous return, pulmonary blood volume and hydrostatic pressure. The elevated hydrostatic pressure in pulmonary vessels leads to pulmonary edema. ${ }^{10}$

Young healthy individuals, especially young male athletes, are at increased risk perioperatively. ${ }^{10}$ They can generate large enough negative intrathoracic pressures to induce the pathophysiologic changes described earlier. Other risk factors during surgery include anatomically difficult intubation; nasal, oral or pharyngeal surgical site of pathology; obesity with obstructive sleep apnea; short neck; and acromegaly. ${ }^{6,8}, 10$ The hazard ratios of the various risk factors have not been reported.

\footnotetext{
Box 1: Causes of postobstructive pulmonary edema

Type I

- Postextubation laryngospasm

- Epiglottitis

- Croup

- Choking/foreign body

- Strangulation

- Hanging

- Endotracheal tube obstruction

- Laryngeal tumour

- Goitre

- Mononucleosis

- Postoperative vocal cord paralysis

- Migration of Foley catheter used to tamponade epistaxis

- Near drowning

- Intraoperative direct suctioning of endotracheal tube adapter

Type II

- Post-tonsillectomy/adenoidectomy

- Post-removal of upper airway tumour

- Choanal stenosis

- Hypertrophic redundant uvula

Reproduced, with permission, from Van Kooy MA, Gargiulo RF. Postobstructive pulmonary edema. Am Fam Physician 2000;62:401-4.
}

\section{How does postobstructive pulmonary edema present?}

Postobstructive pulmonary edema usually presents with rapid onset of acute respiratory failure, including dyspnea and tachypnea. Types I and II share similar clinical presentations, including paradoxical ventilation, pink frothy sputum and severe agitation, but stridor is specific for type I. Rapid onset of pulmonary edema after acute airway obstruction followed by rapid resolution of radiologic and echocardiographic features within a 24-hour period establishes the diagnosis. ${ }^{10}$ Other causes of pulmonary edema, including aspiration pneumonitis, iatrogenic volume overload, and cardiogenic or neurogenic pulmonary edema, must first be excluded.

Our patient's presentation was characterized by rapid resolution of patchy opacities after endotracheal intubation and recurrent early acute respiratory distress after extubation. Cardiogenic pulmonary edema and virus-related acute myocarditis were excluded, based on a normal coronary arteriogram and negative results of virus serological tests. In our patient, recurrent episodes of type I postobstructive pulmonary edema appeared to be caused by tracheomalacia involving a substernal goitre. We drew this conclusion because she was weaned from the ventilator, and extubation was successful after thyroidectomy.

\section{Is this condition treated differently from other causes of pulmonary edema?}

Treatment of postobstructive pulmonary edema is different from other causes of pulmonary edema, especially with regard to the use of diuretics. Treatment depends on severity of symptoms, but is similar for types I and II. A systematic review suggests management commonly includes supportive measures, positive pressure ventilation, diuretics and steroids $;{ }^{10}$ however, there is limited support for the use of diuretics and steroids in this condition. Supportive measures include maintaining a patent airway and adequate oxygen supplement with high fraction of inspired oxygen via continuous positive airway pressure, or intubation and venti lation with positive end-expiratory pressure, especially in severe cases. The recommended initial positive end-expiratory pressure level is 5$10 \mathrm{~cm} \mathrm{H}_{2} \mathrm{O} .^{10}$

Diuretics have been used in this condition; however, their role remains controversial. Diuretics may worsen hypovolemia and hypoperfusion in patients experiencing postobstructive pulmonary edema after surgery. ${ }^{7,11}$ Although steroids are thought to reduce the high negative pressures 
related to the physical damage of alveoli and capillaries, their role is uncertain. ${ }^{10}$

The role of inhaled $\beta$-agonists is limited in upper airway obstruction. However, in postobstructive pulmonary edema, $\beta$-agonists may accelerate the rate of alveolar fluid clearance and facilitate regression of pulmonary edema via increased active cation transport. ${ }^{12}$ Heliox therapy is most commonly used for management of postextubation stridor and asthma exacerbations. The lack of adverse effects and rapid onset suggest that a trial of heliox may be reasonable in these patients $i^{13}$ however, further study is required before definitive recommendations can be made.

\section{Can postobstructive pulmonary edema be prevented?}

Early recognition of patients at risk for upper airway obstruction is important so that preventive measures may be started. Because laryngospasm in perioperative patients is the most common cause, some case series and reviews have suggested adequate anesthesia depth, early relief of laryngospasm with neuromuscular blockers, waking the patient fully before extubation and use of a bite block. ${ }^{7,11}$ One case series emphasizes the importance of correcting underlying pathology, such as neck masses, as in our patient. ${ }^{7}$ For goitres in particular, surgical treatment is generally recommended for those that cause obstructive symptoms and have substernal extension. ${ }^{14}$

\section{What is the relation between goitres and postobstructive pulmonary edema?}

Compression syndromes caused by substernal goitres involve adjacent anatomic structures. The tracheal manifestations of substernal goitre compression are variable, including irritable cough, dyspnea, tracheomalacia and right-sided congestive heart failure secondary to persistent hypoxia. Tracheal compression resulting from chronic respiratory distress and concurrent upper respiratory infections may lead to acute respiratory failure. ${ }^{15}$

We are aware of only 6 instances of postobstructive pulmonary edema associated with thyroid tumours reported in the literature. ${ }^{716-20}$ The features of these cases, including ours, are described in Appendix 1, available at www.cmaj.ca/lookup /suppl/doi:10.1503/cmaj.120256/-/DC1. The causes included 3 cancers ( 2 primary and 1 metastatic) and 4 benign goitres. All of the cases involved surgical intervention. Two of the cases involved pregnant women. The altered physiology of pregnancy, including increases in plasma volume by $50 \%$ and red cell mass by $18 \%$ to $30 \%$, makes the development of pulmonary edema more likely. ${ }^{16,18}$ After acute therapy for pulmonary edema, the symptoms of all patients resolved within 36 hours.

\section{References}

1. Moore RL, Binger CA. The response to respiratory resistance: a comparison of the effects produced by partial obstruction in the inspiratory and expiratory phases of respiration. $J$ Exp Med 1927;45:1065-80.

2. Oswalt CE, Gates GA, Holmstrom MG. Pulmonary edema as a complication of acute airway obstruction. JAMA 1977;238: 1833-5.

3. Ringold S, Klein EJ, Del Beccaro MA. Postobstructive pulmonary edema in children. Pediatr Emerg Care 2004;20:391-5.

4. Guffin TN, Har-el G, Sanders A, et al. Acute postobstructive pulmonary edema. Otolaryngol Head Neck Surg 1995;112:235-7.

5. Van Kooy MA, Gargiulo RF. Postobstructive pulmonary edema. Am Fam Physician 2000;62:401-4.

6. Deepika K, Kenaan CA, Barrocas AM, et al. Negative pressure pulmonary edema after acute upper airway obstruction. J Clin Anesth 1997;9:403-8.

7. Koh MS, Hsu AA, Eng P. Negative pressure pulmonary oedema in the medical intensive care unit. Intensive Care Med 2003; 29:1601-4.

8. Lorch DG, Sahn SA. Post-extubation pulmonary edema following anesthesia induced by upper airway obstruction. Are certain patients at increased risk? Chest 1986;90:802-5.

9. Fremont RD, Kallet RH, Matthay MA, et al. Postobstructive pulmonary edema: a case for hydrostatic mechanisms. Chest 2007;131:1742-6.

10. Udeshi A, Cantie SM, Pierre E. Postobstructive pulmonary edema. J Crit Care 2010; 25:508.e1-5.

11. McConkey PP. Postobstructive pulmonary oedema - a case series and review. Anaesth Intensive Care 2000;28:72-6.

12. Matthay MA, Fukuda N, Frank J, et al. Alveolar epithelial barrier. Role in lung fluid balance in clinical lung injury. Clin Chest Med 2000;21:477-90.

13. Berkenbosch JW, Grueber RE, Graff GR, et al. Patterns of helium-oxygen (heliox) usage in the critical care environment. J Intensive Care Med 2004;19:335-44.

14. Schwartz SI, Brunicardi FC. Schwartz's principles of surgery. 9th ed. New York (NY): McGraw-Hill; 2010. p. 1866.

15. Anders HJ. Compression syndromes caused by substernal goitres. Postgrad Med J 1998;74:327-9.

16. Stradling JR, Bolton P. Upper airways obstruction as cause of pulmonary oedema. Lancet 1982;1:1353-4

17. Leatherman JW, Schwartz S. Pulmonary edema due to upper airway obstruction. South Med J 1983;76:1058-60.

18. Lagler U, Russi E. Upper airway obstruction as a cause of pulmonary edema during late pregnancy. Am J Obstet Gynecol 1987;156:643-4.

19. Ikeda H, Asato R, Chin K, et al. Negative-pressure pulmonary edema after resection of mediastinum thyroid goiter. Acta Otolaryngol 2006;126:886-8.

20. Schaffer SA, Manji RA, Kirkpatrick I, et al. Negative pressure pulmonary edema in the coronary care unit. Can J Cardiol 2008; 24:e58-9.

Affiliations: From the Departments of Internal Medicine (Liu), General Surgery (Shih) and Chest Medicine (Chen), Tri-Service General Hospital, National Defense Medical Center, Taipei, Taiwan

Contributors: All of the authors contributed substantially to the concept, drafting and revision of the manuscript, and approved the final version submitted for publication. 